\title{
Differentiation operation in the wave equation for the pseudospectral method with a staggered mesh
}

\author{
Zhixin Zhao ${ }^{1}$, Jiren $\mathrm{Xu}^{1}$, and Shigeki Horiuchi ${ }^{2}$ \\ ${ }^{1}$ Institute of Geology, Chinese Academy of Geological Science, Beijing 100037, China \\ ${ }^{2}$ National Research Institute for Earth Science and Disaster Prevention (NIED), Science and Technology Agency, \\ 3-1 Tennodai, Tsukuba-shi, Ibaraki-ken 305-0006, Japan
}

(Received March 31, 2000; Revised March 1, 2001; Accepted March 2, 2001)

\begin{abstract}
In the present analysis we introduced a calculation strategy of the staggered grid differentiation by using the real value FFT and real inverted FFT for the pseudospectral method and applied the technique to seismic wave simulation. The calculation method introduced here is one third faster on average than the traditional differentiation method by using the complex FFT. The introduced differentiation strategy is very efficient in economy. For example we apply the staggered grid differentiation by real valued FFT to the simulation of seismic wave propagation in inhomogeneous medium. The results show the validity of the present method.
\end{abstract}

\section{Introduction}

The pseudospectral (PS) method was employed in the calculations for fluid dynamics by Orszag (1972). The method has also been employed widely in the simulations of acoustic wave and elastic wave equations because of its high accuracy and efficiency in use of the computer memory compared with the other discrete numerical method (Kosloff and Baysal, 1982; Fornberg, 1987; Reshef et al., 1988). The time differentiation in the PS method is generally performed by using a second order centered differential operator same as that in the finite difference method (FD) in the computation of synthetic seismograms. The spatial partial differentiation scheme of PS method, however, is different from that of the finite difference method. The spatial differentiation is performed through the operators of a pair of discrete Fourier transform and discrete inverse Fourier transform in the PS method (Ozdenvar and McMechan, 1996; Furumura et al., 1998; Zhao and Kubota, 1999). The Hartley transform is also applied for the spatial differentiation in seismic simulation to improve the speed of differentiation operation (Saatcilar and Ergintav, 1991; Zhou, 1992). The Fourier transform of a discrete real sequence for a seismic wave simulation can be performed by a complex FFT in the PS method. It also can more efficiently be performed by a real FFT in the traditional PS calculation in which the interested study domain is meshed with a centered mesh scheme (Fig. 1(a)). We call the meshed scheme as conventional meshed scheme in order to distinguish it from the staggered grid scheme (Fig. 1(b)). In conventional meshed scheme the relative physical variables are generally all sampled at the identical discretized nodes. The spatial derivatives of the variables are evaluated at the same nodes.

Copy right $(\mathrm{C})$ The Society of Geomagnetism and Earth, Planetary and Space Sciences (SGEPSS); The Seismological Society of Japan; The Volcanological Society of Japan; The Geodetic Society of Japan; The Japanese Society for Planetary Sciences.
A different discrete grid scheme, the staggered grid strategy, has been presented in the spatial numerical differentiation operator because of the more physical and accurate nature. Virieux (1984 and 1986) applied the staggered grid scheme in the FD method. Chen (1996) and Witte (1989) introduced the staggered grid scheme in the PS method to avoid the loss of the Nyquist component. Figure 1(b) shows the sampled parameters for a 2-D $S H$ wave propagation of the elastic dynamic problem. In the staggered grid case, some components of variables are sampled and evaluated at the discretized nodes. The other components (even for the components of identical variable), however, are sampled half-way between the discreteized nodes (taken 1/2 simple shift in the Cartesian coordinates axes). The spatial differentiation is evaluated at the midway between grid points for a problem of the staggered grid scheme. For example in Fig. 1(b), the velocity of media $V_{y}$ is defined at the node of grid $(n, m)$, the stresses $\tau_{x y}$ and $\tau_{y z}$ are defined at the halfway between the nodes in horizontal and vertical directions, respectively. The Lame constant $\mu$ and the density $\rho$ can be defined at the center of grid. The numerical scheme of 2-D SH wave equation can be expressed as follows,

$$
\rho_{n, m} \dot{v}_{y n, m}=\partial \dot{\tau}_{x y n+1 / 2, m} / \partial x+\partial \dot{\tau}_{y z n, m+1 / 2} / \partial z+f_{y} .
$$

Such differentiation operators of the first and second terms in the right hand side of (1) should be evaluated at the midway between the nodes, $(n+1 / 2) \Delta x$ and $(m+1 / 2) \Delta z$, respectively.

The differential operator can be performed by the complex FFT. We, however, prefer to present a strategy for the staggered grid differential operator by using the real valued FFT and inverse FFT in the following section. The method is called as "RFFT staggered differentiation" for short. 


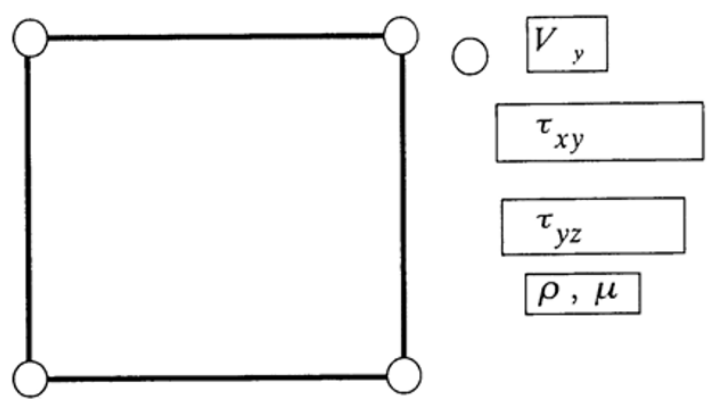

(a)
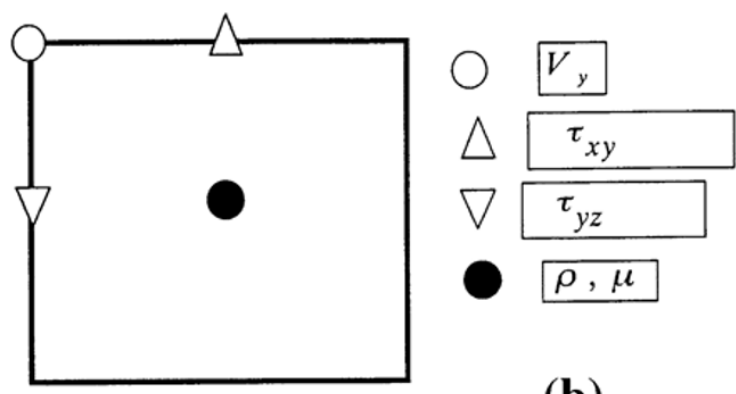

(b)

Fig. 1. The sampled parameters for a 2-D $S H$ problem. a) The conventional grid scheme; b) The staggered grid scheme. $V_{y}$ : velocity; $\tau_{x y}$ and $\tau_{y z}$ : stresses; $\mu$ : Lame constant; $\rho$ : density.

\section{Expansion of Fourier Series}

After the analysis by Furumura et al. (1998) a finite Fourier series expansion of a real sequence $f(n \Delta x)(n=0,1, \ldots$, $N-1)$ can be written as

$$
\begin{aligned}
f(n \Delta x)= & \sum_{l=0}^{N / 2} A(l \Delta k) \cos (2 \pi n l / N) \\
& +\sum_{l=1}^{N / 2-1} B(l \Delta k) \sin (2 \pi n l / N),
\end{aligned}
$$

where $A(l \Delta k)$ and $B(l \Delta k)$ are the coefficients of cosine and sine, they are,

$$
\begin{aligned}
A(l \Delta k)= & \frac{1}{N} \sum_{n=0}^{N-1} f(n \Delta x) \cos (2 \pi n l / N), \\
& l=0, N / 2 \\
A(l \Delta k)= & \frac{2}{N} \sum_{n=0}^{N-1} f(n \Delta x) \cos (2 \pi n l / N), \\
& l=1,2, \ldots, N / 2-1
\end{aligned}
$$

and

$$
\begin{gathered}
B(l \Delta k)=\frac{2}{N} \sum_{n=1}^{N-1} f(n \Delta x) \sin (2 \pi n l / N), \\
l=1,2, \ldots, N / 2-1
\end{gathered}
$$

where $\Delta k=2 \pi / N \Delta x$ is the interval of discrete spatial wavenumber.

\section{Complex Differentiation for the Staggered Grid Case}

As respect to complex differential operator in the conventional mesh scheme, coefficients of the Fourier transform (FT) of real series (e.g. $V$ or $\tau$ in (1)) are conjugate symmetric about the Nyquist term and the imaginary parts of Nyquist terms are zero. In order to obtain a real differential result for original real series, the FT coefficients multiplied by wavenumber $l \Delta k$ with imaginary number $i$ also should be a conjugate symmetric one and the imaginary part of Nyquist term should be zero (real coefficient already becomes zero). So the imaginary part of Nyquist term forced to be zero in the inverse Fourier transform. The Nyquist information is lost.

As the spatial differentiation of variable $V$ and $\tau$ in the wave problem in (1) are operated in a staggered meshed scheme in the PS method, derivatives in (1) are evaluated at the position of midway between the discretized grids $(n+$ $1 / 2) \Delta x$, shifting a $1 / 2$ sample. The expression of complex differentiation is as follows (Witte, 1989):

$$
\begin{aligned}
d^{+} f(x) / d x= & d f((n+1 / 2) \Delta x) / d x \\
= & \sum_{l=0}^{N-1} i l \Delta k e^{i \pi l / N} F(l \Delta k) e^{i 2 \pi n l / N} \\
& n=0,1,2, \ldots, N-1
\end{aligned}
$$

where $F(l \Delta k)$ is the complex FT of $f(n \Delta x)$.

It can be seen that spectral differential operator in the conventional mesh scheme, $i l \Delta k$, has been replaced by the staggered spectral differential operator in (6), il $\Delta k \exp (i \pi l / N)$. The Nyquist wavenumber (as $l=N / 2$ ) in (6) is no longer equal to zero, being $(-\pi / \Delta x)$, a purely real. So the numerically computed derivative is also purely real. The Nyquist information about the original function is retained throughout the process. Because Nyquist information is preserved and used, the staggered differential operators are more local. The nonzero Nyquist term makes the differentiation operator (6) more stable in the PS method. It is different from that in the conventional mesh scheme. The differentiation can also be performed by using complex FFT through transforming the real sequence into complex one in which the imaginary components are filled up with zero values, or separating the real sequence in to real and imaginary parts of a complex one. Then it is transformed back to the physic domain by using the complex inverse FFT.

\section{Real Fourier Differentiation Method for the Staggered Grid Case}

We, however, try to introduce a more efficient scheme for the staggered grid differentiation in wave problem as in (1) by using the real valued FFT here. The development of the real valued FFT operator in present analysis very closely follows the analysis presented by Furumura et al. (1998) for conventional meshed schemes. We extend the application of these operators to the staggered grid system now. The differentiation of a discrete real sequence $f(n \Delta x)$ at the midway between the nodes $(n+1 / 2) \Delta x$ by using the real FFT (2) and real inverse FFT can be written as follows, 


$$
\begin{aligned}
d^{+} f(x) / d x \\
=\frac{d}{d x} f((n+1 / 2) \Delta x) \\
=\sum_{l=1}^{N / 2-1}(l \Delta k) B(l \Delta k) \cos (2 \pi(n+1 / 2) l / N) \\
\quad+\sum_{l=0}^{N / 2}(-l \Delta k) A(l \Delta k) \sin (2 \pi(n+1 / 2) l / N),
\end{aligned}
$$

where coefficients $A$ and $B$ and $(l \Delta k)$ are same as those in (3), (4) and (5). We also note that $l \Delta k=0, B(l \Delta k)=0$, and $\sin (2 \pi n l / N)=0$ as $l=0$ or $l=N / 2$.

After the proper trigonometric operators, Eq. (7) becomes as follows,

$$
\begin{aligned}
\frac{d}{d x} f((n & +1 / 2) \Delta x) \\
= & \sum_{l=0}^{N / 2}(l \Delta x)(\cos (\pi l / N) B(l \Delta k) \\
& -\sin (\pi l / N) A(l \Delta k)) \cos (2 \pi n l / N) \\
& +\sum_{l=1}^{N / 2-1}(-l \Delta k)(\cos (\pi l / N) A(l \Delta k) \\
& +\sin (\pi l / N) B(l \Delta k) \sin (2 \pi n l / N) .
\end{aligned}
$$

We assume

$$
\begin{aligned}
A 1= & (l \Delta k)(\cos (\pi l / N) B(l \Delta k) \\
& -\sin (\pi l / N) A(l \Delta k)) \\
B 1= & (-l \Delta k)(\cos (\pi l / N) A(l \Delta k) \\
& +\sin (\pi l / N) B(l \Delta k)) .
\end{aligned}
$$

Equation (8) is simplified as follows

$$
\begin{aligned}
& \frac{d}{d x} f((n+1 / 2) \Delta x) \\
&=\sum_{l=0}^{N / 2} A 1(l \Delta k) \cos (2 \pi n l / N) \\
&+\sum_{l=1}^{N / 2-1} B 1(l \Delta k) \sin (2 \pi n l / N)
\end{aligned}
$$

This is what we are interested in. It can be seen that the staggered grid differentiation (8) or (10) as employing in the PS method can be easily performed by using the real FFT and inverse FFT similarly to the conventional mesh differentiation. The procedure for the above staggered grid differentiation is summarized as follows. We first obtain the coefficients $A(l \Delta k)$ and $B(l \Delta k)$ of real data $f(n(\Delta x))$ ( $n=0,1, \ldots, N-1)$ according to the formulas (3), (4) and (5). Then we calculate the $A 1(l \Delta k)$ and $B 1(l \Delta k)$ coefficients of cosine and sine of (10) according (9). The staggered grid differentiation can be calculated with implementing the real inverse FFT for (8) or (10).

We perform the staggered differentiation (8) or (10) by using the real FFT one third faster than by using the complex FFT in (6). So it has lower economic cost to using the differentiation of (10) in PS method. It is obviously seen that the Nyquist coefficient term is also retained (the second term of the right side of (8)). It is nonzero, equal to $(-\pi / \Delta x) A(\Delta k N / 2)$. So the stability of the differentiation of (8) by using real FFT is same as that by using the complex FFT in (6).

\section{Comparison for Real FFT Staggered Operator and Cagniard-De Hoop Method}

To demonstrate the accuracy of the algorithm presented in this analysis, we compared the calculation result by the real valued FFT differential operators in the staggered grid system presented in the analysis with the result from the analytic result of Cagniard-De Hoop method (Aki and Richards, 1980) for a 2-D $S H$ problem in half space with a line source as shown in Fig. 2(a). Figure 2(a) shows a homogenous half space model. The source directional force acting in the $y$ direction is taken as a Gaussian shape with a Ricker wavelet source time function having a peak frequency of 25 $\mathrm{Hz}$. The time step is 0.001 seconds. In the numerical simulation the $2-\mathrm{D}$ model is sampled with $128 \times 128$ points at $20 \mathrm{~m}$ spacing in both horizontal and vertical directions. The absorbing boundary condition (Cerjan et al., 1985) is applied on the lateral and bottom edges of the spatial grid in treating the boundary condition. The free-surface condition is approximated by including N-points zero shear velocity above the upper surface of the model in vertical derivatives of stress. The symmetric differentiation method by Furumura and Takenaka (1992) is applied for vertical differentiation of displacement to stabilize the derivatives near the surface nodes. Locations B, C, D and E in Fig. 2(a) are receivers. We will demonstrate calculated waveforms at those locations by the RFFT staggered differentiation method with that calculated by the analytic Caganiard-De Hoop method. Figure 2(b) shows the waveform comparisons between results from the above two different methods. Waveforms obtained from RFFT staggered differentiation method (solid circles) and from Cagnard-De Hoop method (thin solid line) are all coincide well for location B, C, D and E, especially in the first direct phase. The difference can almost not be distinguished in the waveforms. Small difference can be seen between the two kinds of waveforms in the later reflection phase from surface (somewhat easily seeing in case at point B). Results in Fig. 1(b) suggest that accuracy of the real valued FFT differential technique in staggered system is comparable with the Cagniard-De Hoop technique. The accuracy of the RFFT staggered differentiation operator in the staggered system is satisfactory.

\section{Example}

We apply the staggered grid differentiation of (10) for the PS method to simulate the $S H$ wave propagation in the model of the heterogeneous medium as shown in Fig. 3. There is a thrust fault with a thrust angle of 45 degrees in the model. The velocity of $S$ wave and the density are $1000 \mathrm{~m} / \mathrm{s}$ and $2.1 \mathrm{~g} / \mathrm{cm}^{3}$ in region I (being the upper layer of the foot wall of thrust fault), $2000 \mathrm{~m} / \mathrm{s}$ and $2.5 \mathrm{~g} / \mathrm{cm}^{3}$ in region II, respectively. The 2 -D model is sampled with $256 \times 128$ points in the horizontal and dip direction at $50 \mathrm{~m}$ spacing in both directions. The thickness of region I is $2 \mathrm{~km}$. The surface node (or node line) of the thrust fault plane is located at $6 \mathrm{~km}$ away from the left end of the model (corresponding to the 120th grid in Fig. 4). The time interval is taken as 0.005 seconds. The Herrmann's 


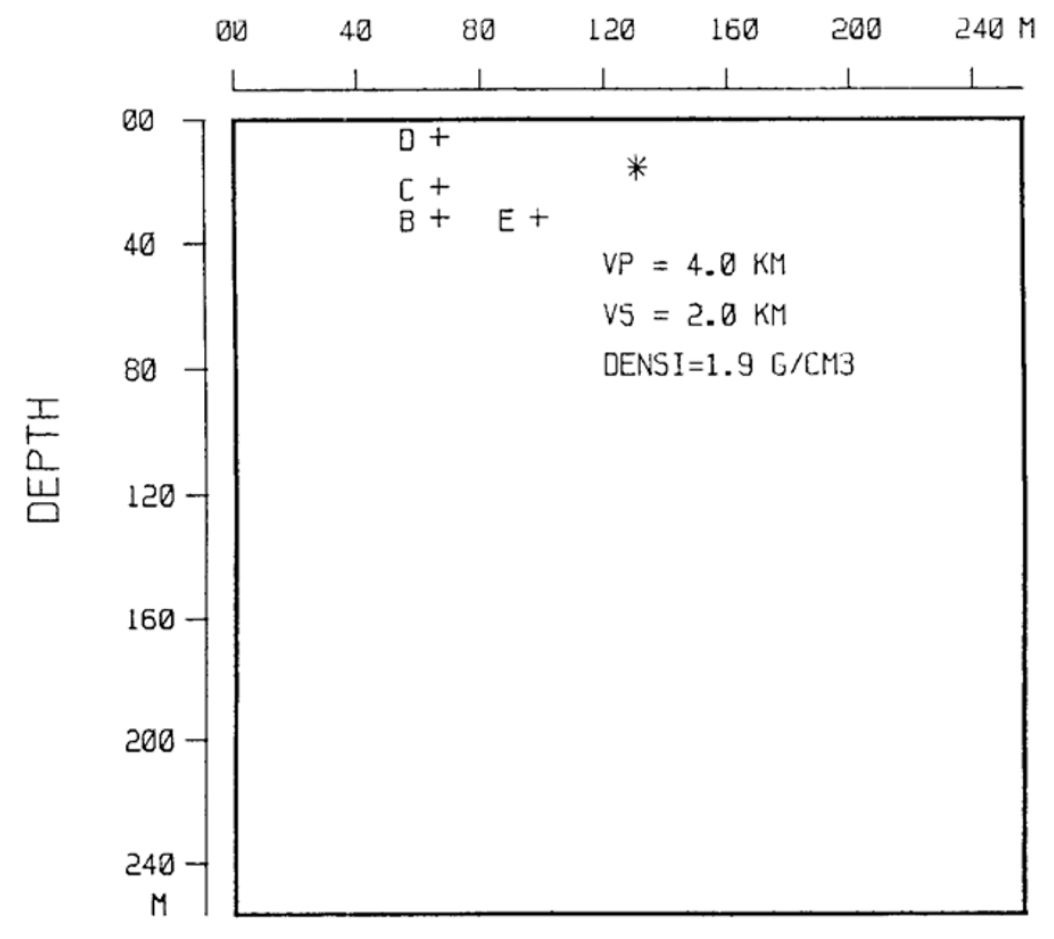

(a)
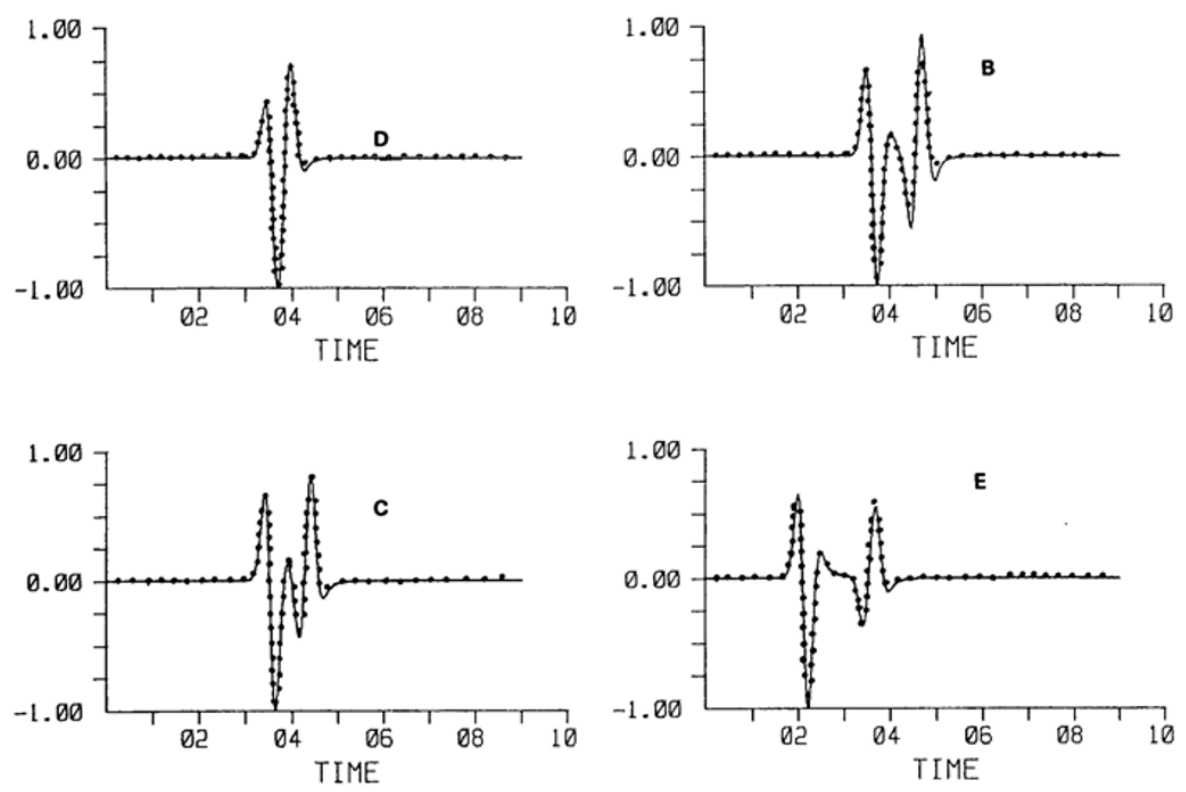

(b)

Fig. 2. Comparisons for waveforms calculated from the real valued FFT differential operator in the staggered system presented by this analysis and the Cagniard-De Hoop method. a) Half space homogeneous model for a 2-D SH problem; asterisk for line source; B, C, D and E are receivers. b) Waveform comparisons; solid circles for the waveform calculated from the RFFT staggered differentiation method by this paper; thin solid line for waveform from the Cagniard-De Hoop method. The unit of time is 0.1 seconds.

time function (Harrmann, 1979) having 0.4 seconds width with a spatial Guassian shape is used in the analysis taking as the source function. The surface condition and boundary condition in calculation schemes are treated similarly as those used in Section 5.

Figure 3 shows the simulated snapshots at time from 2.0 to 5.0 seconds too. The shots clearly show the propagation characteristics of $S H$ seismic waves in the heterogeneous medium. In shot at 3.0 seconds the wave in region II (the higher velocity region) transmits over the bottom of region I and propagates upwardly in region I (the lower velocity region). The spherical wave in region II is refracted at the bottom and propagates with a plane wavefront in region I in the shots at 4.0 and 5.0 seconds. In other way the wave 

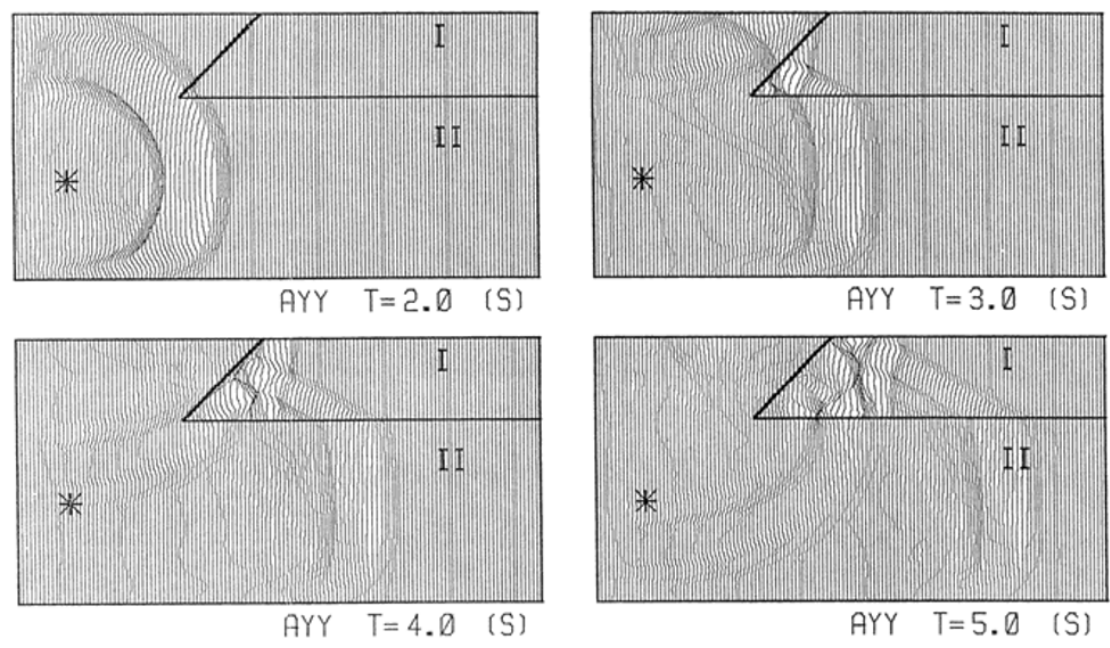

Fig. 3. Heterogeneous velocity structure model with a thrust fault having thrust angle of 45 degrees and snapshots of simulation. Thickness of region I is $2 \mathrm{~km}$. Surface node of the fault plane is $6 \mathrm{~km}$ away from the left end corresponding to the 120 th grid in Fig. 4 . The velocity of $S$ wave and the density are $1000 \mathrm{~m} / \mathrm{s}$ and $2.1 \mathrm{~g} / \mathrm{cm}^{3}$ in the region I, $2000 \mathrm{~m} / \mathrm{s}$ and $2.5 \mathrm{~g} / \mathrm{cm}^{3}$ in the region II, respectively. The asterisk for the line source.

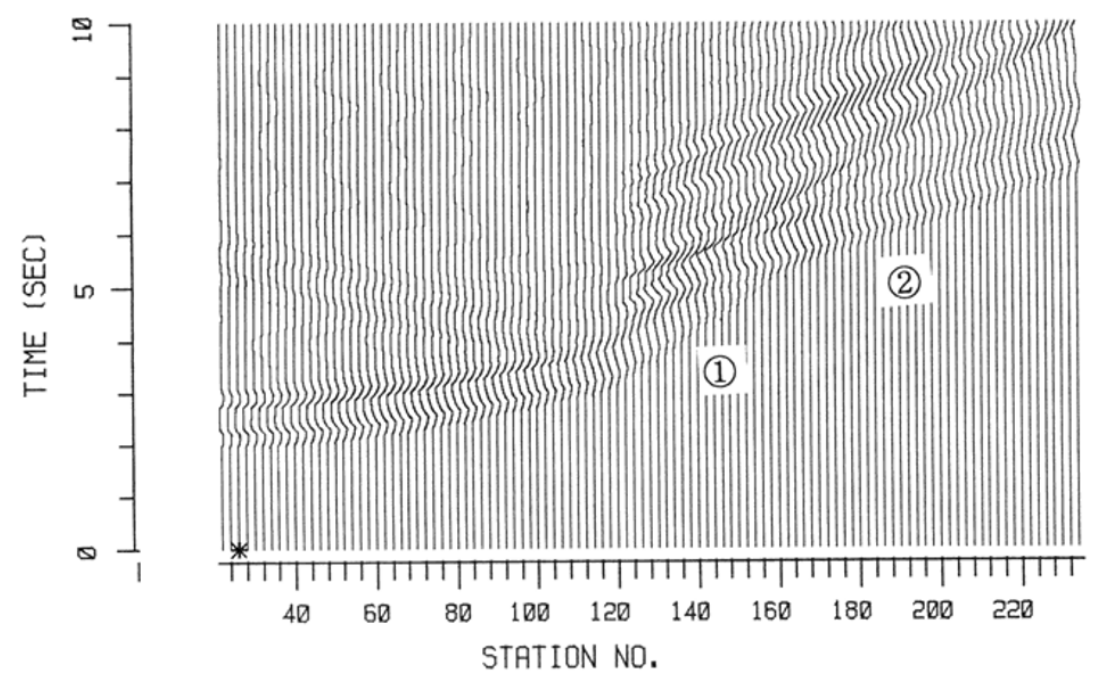

Fig. 4. The waveforms at sites on the surface in Fig. 3. Abscissa for number of stations (record sites), their distances away from the left end are equal to the station number multiplying by the grid spacing of $50 \mathrm{~m}$. (1) for the body wave phase, (2) for the secondly surface wave phase.

in region II arrives at the free surface of region II fast and is reflected at the surface (seeing the left surface in Fig. 3). We can also see that the wave passes through the fault plane, enters the region I in the shot of 3.0 seconds and propagates along the horizontal direction in region I (seeing shots of 4.0 and 5.0 seconds). We called the wave propagating along horizontal layer (region I) as the secondly surface wave here. The secondly surface wave arrives in the left surface of region I before the upward waves do (seeing the shot of 4.0 seconds). The interference formed of the upward body wave phase and the secondly surface wave propagating along the surface from region II to region I can be seen at the shots of 4.0 and 5.0 seconds. The interference can usually result in the peak of the ground motion that causes large seismic mitigation (Zhao and Kubota, 1997). Irikura et al. (1999) collected some reports on wave interference in the ground motion. Pitarka et al. (1998) and Kawase et al. (1998) also reported the relationship between the peak ground motion and the secondly surface wave in the simulations of the 1995 Hyogoken Nanbu earthquake by the FD method. We also can see that the reflected wave from the surface in region II then enters region I along the fault plane and the bottom of region I.

Figure 4 shows the waveforms at the sites on the surface in Fig. 3. The horizontal distances of sites are equal to the station number multiplying by $50 \mathrm{~m}$, the grid spacing. The direct wave and weak reflected phase can be identified on the waveforms at site numbers less than 120 (the surface node of thrust fault plane) in region II (Fig. 3). Waveforms are simple too. Their velocity is $2.0 \mathrm{~km} / \mathrm{s}$ which is the $S$ wave velocity of region II in Fig. 3. For the waveforms at site number larger than 120 (in region I in Fig. 3), the traveltime curve picked from the first arrival phases between about the 120th and the 160th sites gets steep (around the symbol (1) region). It implies the velocity of waves becomes slow. The velocity 
is equal to $1.0 \mathrm{~km} / \mathrm{s}$, the same as that of region I in Fig. 3. The phase may be the second surface wave propagating along the layer I in Fig. 3 as mentioned above. The traveltime curve picked from the first arrivals for the site number larger than 160 gets even (around region of symbol (2)), the velocity of wave gets fast. The velocity is near $2.0 \mathrm{~km} / \mathrm{s}$ which is the apparent velocity of the upward waves with plane wavefront (seeing shots of 4.0 and 5.0 seconds in Fig. 3). Some later phases also can be seen in the waveforms in Fig. 4.

\section{Discussion and Conclusion}

We introduced a calculation strategy of the staggered grid differentiation by using the real value FFT and inverse FFT for the PS method of the seismic wave propagation in the present analysis. The calculation by using the RFFT staggered differentiation method is one third faster than that by using the traditional complex FFT in the staggered meshed scheme. The Nyquist coefficient term is also retained, and the differentiation is more stable. The differentiation calculation strategy introduced in the present analysis is very efficient in economy. In the example for simulation of $\mathrm{SH}$ wave propagation, we can clearly see the direct wave and reflected wave in region II in Figs. 3 and 4. In the low velocity region I we can clearly observe the two transmitting waves, the secondly surface wave from the thrust fault plane and the upward body wave from the bottom of region I. The phenomena mentioned above can also be identified in the snapshots in Fig. 3. The results in Figs. 3 and 4 suggest that the RFFT staggered differentiation operator for the PS method is applicable for seismic simulation.

The differential operators in the staggered grid system by using real valued FFT presented in the analysis is already applied to a 2-D $S H$ problem. We think that the RFFT staggered differentiation operator can also be extended to more complete wave propagation systems in 2-D or 3-D problem as an effective method.

Acknowledgments. The authors are very grateful to the two anonymous reviewers for their valuable comments to improve this paper. We also express our heartfelt thanks to professor M. Kikuchi in University of Tokyo for the helpful suggestions and comments. This study is financially supported by the research projects 2000444 and 2000445 of Ministry of Land and Source of China.

\section{References}

Aki, K. and P. G. Richards, Quantitative seismology theory and methods, pp. 224-243, W. H. Freeman and Company, San Francisco, U.S.A., 1980.
Cerjan, C., D. Kosloff, R. Kosloff, and M. Reshef, A nonreflecting boundary condition for discrete acoustic and elastic wave equation, Geophysics, $\mathbf{5 0}$, 705-708, 1985.

Chen, H. W., Staggered grid pseudospectral simulation in viscoacoustic wavefield simulation, J. Acoust. Soc. Am., 100, 120-131, 1996.

Fornberg, B., The pseudospectral method: Comparisons with finite difference for the elastic wave equation, Geophysics, 52, 483-501, 1987.

Furumura, T. and H. Takenaka, A stable method for numerical differentiation of data with discontinuities at end-points by means of fourier transform-symmetric differentiation, Geophys. Expl., 45, 303-309, 1992 (in Japanese).

Furumura, T., B. L. N. Kennett, and H. Takenaka, Parallel 3-D pseudospectral simulation of seismic wave propagation, Geophysics, 63, 279-288, 1998.

Herrmann, R. B., SH-wave generation by dislocation sources-A numerical study, BSSA, 69, 1-15, 1979.

Irikura, K., K. Kudo, H. Okada, and T. Sasatani, The effect of surface geology on seismic motion, in Chap. 3, simultaneous simulation of Kobe, A.A. Balkema, 1594 pp., Rotterdam Press, Netherlands, 1999.

Kawase, H., S. Matsushima, R. W. Graves, and P. G. Somervelle, Threedimensional wave propagation-The cause of the damage belt during the 1995 Hyogo-ken Nanbu earthquake, Zishin, 50, 431-449, 1998.

Kosloff, R. and E. Baysal, Forward modeling by a Fourier method, Geophysics, 42, 1402-1412, 1982.

Orszag, S. A., Comparison of pseudospectral and spectral approximation, Stud. Appl. Math., 51, 253-259, 1972.

Ozdenvar, T. and G. A. McMechan, Causes and reduction of numerical artefacts in pseudo-spectral wavefield extrapolation, Geophys. J. Int., 126, 819-828, 1996.

Pitarka, A., K. Irikura, T. Iwata, and H. Sekikuchi, Three-dimensional simulation of the near-fault ground motion for the 1995 Hyogo-ken Nanbu (Kobe), Japan, earthquake, BSSA, 88, 428-440, 1998.

Reshef, M., D. Kosloff, M. Edwards, and C. Hsiung, Three-dimensional elastic modeling by the Fourier method, Geophysics, 53, 1184-1193, 1988.

Saatcilar, R. and S. Ergintav, Solving elastic wave equations with the Hartley method, Geophysics, 56, 247-278, 1991.

Virieux, J., SH wave propagation in heterogeneous media: velocity-stress finite difference method, Geophysics, 49, 1933-1957, 1984.

Virieux, J., p-sv wave propagation in heterogeneous media: velocity-stress finite-difference method, Geophysics, 51, 889-901, 1986.

Witte, D. C., The pseudospectral method for simulating wave propagation, Doctor Thesis, Columbia University, 29 pp., 1989.

Zhao, Z.-X. and R. Kubota, The distribution of strong ground motion from uppermost crustal structure-comparison with disaster from the Hyogoken nanbu earthquake, Proceeding of the 97th SEGJ Conference 7174, The Society of Exploration Geophysicists of Japan, October, 1997, Sapporo, Japan, 1997.

Zhao, Z.-X. and R. Kubota, The empirical Green's function method by using simulated waveforms, in The Effect of Surface geology on Seismic Motion-Recent Progress and New Horizon on ESG Study, Volume 3, edited by K. Irikura, K. Kudo, H. Okata, and T. Sasatani, pp. 1435-1442, A.A. Balkema, Rotterdam Press, Netherlands, 1999.

Zhou, B., On the use of the Hartley transform in geophysical applications, Geophysics, 57, 196-197, 1992.

Z. Zhao (e-mail: zhaozhixin@ccsd.org.cn), J. Xu, and S. Horiuchi 